Revista Iberoamericana, Vol. LXXI, Núm. 210, Enero-Marzo 2005, 165-174

\begin{abstract}
LAS MUJERES TAMBIÉN CUENTAN EN EL ECUADOR: REFLEXIONES SOBRE TRES ANTOLOGÍAS RECIENTES DE NARRADORAS ECUATORIANAS Y EL LUGAR QUE ÉSTAS OCUPAN EN EL IMAGINARIO NACIONAL
\end{abstract}

\author{
POR \\ Michael Handelsman \\ University of Tennessee, Knoxville
}

\title{
REFLEXIONES PRELIMINARES
}

Desde hace mucho tiempo en el Ecuador, se dice con cierta ironía y amargura que publicar allí equivale a seguir inédito. Lamentablemente, aquel dicho apunta a toda una historia editorial que ha condicionado la imagen que se tiene del Ecuador como un país de escritores o, más bien, como un país sin escritores. Con la excepción de Juan Montalvo, Jorge Icaza y Jorge Carrera Andrade, pocos son los nombres que constan con regularidad en las diferentes historias literarias de América Latina. Entre las muchas causas que ayudan a explicar esta ausencia, podemos destacar un sistema ineficiente (por no decir inexistente) de distribución y promoción internas y la falta total de una red internacional capaz de difundir los libros nacionales en el exterior. Es decir, los libros no circulan mayormente ni dentro ni fuera del Ecuador.

Como es de suponer, han existido muchos intentos de promover la literatura ecuatoriana con la publicación de diferentes series de libros clásicos nacionales (y a precios módicos), especialmente desde la fundación de la Casa de la Cultura Ecuatoriana en 1944. Sin embargo, estos proyectos patrocinados, ora por entidades públicas ora por las privadas, han sido fugaces, fragmentados y demasiado esporádicos; todo lo cual pone de relieve la ausencia general de una política cultural consistente, creativa y constante. Las consecuencias perjudiciales de tirajes reducidos, libros perdidos en bodegas húmedas y una distribución de libros principalmente a cargo de los mismos autores, no solamente han sido sentidas por los escritores, sino también por los lectores, muchos de los cuales han llegado a la conclusión de que no hay buena literatura en el Ecuador. De hecho, debido a las dificultades de conseguir los libros nacionales, se ha cultivado toda una mentalidad de negación y autodesprecio respecto de las letras nacionales que, supuestamente, han sido marcadas por atrasos en relación al desarrollo de la literatura del resto de Latinoamérica, y por una escasez de escritores de la calidad de, por ejemplo, Paz, García Márquez, Cortázar, Vargas Llosa, Neruda, Borges, Carpentier, entre muchos otros.

De modo que escribir en el Ecuador sigue considerándose heroico, si no quijotesco $\mathrm{y}$, como se ha de imaginar, ha sido especialmente adverso para toda mujer que ha tratado de incorporarse al mundo literario. Vale recordar que en la década del ochenta del siglo xx, cuando la Editorial El Conejo lanzó una ambiciosa campaña de publicación (cien títulos clásicos nacionales junto con una amplia distribución de los mismos en todo el país 
bajo el lema de "El Ecuador escribe,”) se declaraba categóricamente, en 1983, que el Ecuador "no es un país de escritoras" (Donoso Pareja, "La mujer en la narrativa ecuatoriana,” 4). Aunque la intención del autor de esta afirmación fue la de convocar a más mujeres a tomar la palabra y, así, servir de catalizador más que de detractor, la brevedad del comentario que fue publicado en un periódico local evidenció una falta de matización de conceptos que, por lo tanto, se prestaba a lecturas parciales destinadas a ratificar una larga tradición patriarcal que no contemplaba a la mujer como una real fuerza intelectual y plenamente integrada al imaginario nacional. Es decir, en vista de esta tradición exclusivista, la simplificación del artículo señalado le daba razón a un Ecuador que seguía desvalorando el complejo proceso evolutivo de sus escritoras, mientras ignoraba (o negaba) la emergencica de una nueva manera de aprehender el aporte femenino a la literatura, tanto a nivel nacional como a nivel latinoamericano.

Hemos de insistir que la situación de las escritoras del Ecuador no constituía una experiencia aislada. Conviene recordar que Elaine Showalter había comentado en la misma década del ochenta que, en una segunda etapa de la crítica feminista, se había descubierto que "las escritoras tenían una literatura propia, cuya coherencia histórica y temática, además de su importancia artística, habían sido ofuscadas por los valores patriarcales que dominan nuestra cultura” (6, traducción nuestra). Más aún, la misma Showalter puntualizó:

\begin{abstract}
Enfocarse en la escritura de la mujer como un campo específico de investigación, además, condujo a una recuperación masiva y a una nueva lectura de la literatura escrita por las mujeres de todas las naciones y de todas las épocas. Mientras se descubría a centenares de escritoras perdidas, y cartas y diarios salían a la luz y nuevas biografías literarias exploraban las relaciones entre el talento individual de la mujer y la tradición literaria, las continuidades presentes en la escritura de la mujer se volvieron claras por primera vez. (6, traducción nuestra)
\end{abstract}

En efecto, nuestro reparo a la afirmación ya citada de Donoso Pareja-0, por lo menos, a las posibles lecturas simplistas de la misma- ha de comprenderse como una advertencia frente al peligro de leer pasiva y acríticamente que "El Ecuador escribe”, junto con el Ecuador "no es un país de escritoras” y, así, descontextualizar no sólo las tradiciones literarias de las escritoras ecuatorianas, sino las de todos los escritores del país. No estará de más recordar que al entrar la crítica feminista en una tercera etapa, "no se buscaba sólo el reconocimiento de la escritura de la mujer, sino una nueva concepción de los fundamentos mismos de los estudios literarios, una revisión de las premisas teóricas aceptadas sobre el leer y el escribir que han sido basadas estrictamente en las experiencias literarias del hombre” (Showalter 8). Lógicamente, una aceptación ociosa de la noción de que el Ecuador escribe, pero sin sus mujeres, frustraría toda posibilidad de revalorar el lugar que las escritoras ocupan en la historia literaria del país por una parte, y repensar los conceptos mismos de lo que es la historia literaria nacional por otra parte. ${ }^{1}$

${ }^{1}$ La identificación de materiales poco conocidos, pero pertinentes al estudio de las escritoras en la historia literaria del Ecuador, y el intento de introducir nuevos enfoques para revalorar el aporte de las mujeres a dicha literatura, fueron los motivos principales de nuestro estudio, Amazonas y 
Aunque parezca una contradicción, las tres antologías que comentaremos a continuación no apuntan a separatismos, ni pretenden privilegiar una escritura supuestamente femenina (o feminista) por naturaleza. Conviene recordar lo que Donoso Pareja había puntualizado en la introducción a su recopilación de cuentos escritos por mujeres: "De ahí proviene la validez de este libro, la utilidad de esta muestra de lo que han significado y significan las mujeres en el desarrollo del cuento en el Ecuador” (12). Es decir, para aprehender cabalmente la rica historia del cuento ecuatoriano, hace falta leer y analizar el aporte de las mujeres como co-partícipes dentro de la producción cuentística nacional, lo cual pone de relieve la centralidad de las narradoras más que su aparente marginalidad. Si se toma en cuenta que hasta la fecha muy pocos investigadores y lectores, en general, han tenido un conocimiento adecuado sobre el aporte de las ecuatorianas a la literatura y las condiciones socioculturales que lo han marcado, debido a la poca accesibilidad de las obras escritas ya mencionada arriba, se comprenderá el verdadero significado de la publicación de tres antologías en un espacio de apenas cuatro años (19972001). Por primera vez, existe un corpus substancioso de materiales que se presta a lecturas comprehensivas capaces de inspirar nuevas investigaciones y nuevas concepciones de la literatura ecuatoriana vis-à-vis las contribuciones de las mujeres a la misma.

\section{Miguel Donoso Pareja hace justicia a un Reclamo contundente}

Es un poco irónico que la persona que había denunciado en 1983 una ausencia rotunda de narradoras en las letras ecuatorianas haya sido responsable de publicar la primera antología dedicada específicamente a las creadoras y a comprobar la producción valiosa de éstas, tanto por su cantidad como por su calidad. De hecho, y en honor a la verdad, durante los catorce años que separan su reclamo crítico y su Antología de narradoras ecuatorianas, Donoso Pareja había ofrecido varios talleres literarios en el país donde se formaron a algunos de los narradores y narradoras jóvenes más destacados del Ecuador contemporáneo. Si bien es cierto que el objetivo de los talleres no fue el de formar a las mujeres como narradoras propiamente, es también cierto que la actitud críticamente abierta y la rigurosa disciplina de trabajo que caracterizaron el proyecto de Donoso Pareja -y el de otros talleristas que emularon aquel modelo a partir de la década del ochenta-, contribuyeron a crear espacios colectivos desde los cuales todos los aspirantes a ser escritores lograron superar los silencios del aislamiento. Para la mayoría de las mujeres, que tradicionalmente había vivido la escritura como un acto solitario, el nuevo diálogo e intercambio de ideas y prácticas tan patentes en los talleres literarios abrieron oportunidades de aprendizaje y creación que, hasta entonces, le eran virtualmente desconocidas e inalcanzables. Sin duda alguna, este cambio iba a afectar profundamente el desarrollo de

artistas: Un estudio de la prosa de la mujer ecuatoriana, 2 tomos. Guayaquil: Casa de la Cultura Ecuatoriana, Núcleo del Guayas, 1978. De interés especial respecto a la capacidad (y a las realizaciones) de la mujer de crear canales propios de comunicación, pese a la insistencia tradicional de que las ecuatorianas no habían escrito prosa, es el capítulo III, del primer tomo, sobre las revistas hechas por las mujeres desde fines del siglo XIX. 
la producción literaria de las ecuatorianas de fines del siglo xx y, por extensión, el de toda la literatura nacional. ${ }^{2}$

Mientras que la antología de Donoso Pareja contiene veintiocho narradoras, la mayoría de las cuales no comenzó a publicar sino hasta $1980 .^{3}$ Según se lee en la introducción,

Lo que primero podemos observar es que en los primeros 70 años registramos sólo 10 autoras de cuentos, siete de éstas con libros publicados, mientras que en las dos últimas décadas registramos 18 , es decir, virtualmente el doble en menos de la tercera parte del tiempo transcurrido, y prácticamente todas con libros a su haber. Esto se debe, desde luego, al desarrollo cuantitativo y cualitativo, en general, de la narrativa en el país que, como lo subraya Diego Araujo, permite constatar que "el número de novelas casi se duplica desde 1970 en adelante en comparación con las obras editadas durante los veinte años hacia atrás". Asimismo, esto corresponde a las conquistas de la mujer en la sociedad, que se acentúan en las décadas recientes. Cabe puntualizar, además, que la influencia del contexto histórico-social sobre la obra artística en general y la literaria en particular se da a través de un gran número de mediaciones y no de una manera directa. (29-30)

Aunque no es nuestra intención detenernos en comparaciones cuantitativas de los libros publicados y, así, caer en posibles confusiones acerca de la evolución constante de la literatura nacional, sí quisiéramos resaltar las obvias correspondencias entre la producción particular de las mujeres y la del país en general, comprobando una vez más la centralidad de la mujer en la literatura ecuatoriana. Además, prestaremos especial atención a "la influencia del contexto histórico-social” y al "gran número de mediaciones" que Donoso Pareja hace al final de la cita, puesto que pone de relieve la urgente necesidad de problematizar nuestras lecturas (e investigaciones) y, de esta manera, descubrir lo que está detrás de los supuestos silencios.

Anotaremos que Antología de narradoras ecuatorianas pertenece a la Colección Antares de la Editorial Libresa de Quito y, como tal, pretende llegar a un amplio público de lectores, especialmente a los estudiantes de colegio y universidad. Con precios módicos y una primera edición de 3.000 ejemplares, que para un medio como el del Ecuador es excepcional, parece que la antología ha circulado bastante en los principales centros urbanos del país. Según el formato de la colección, que dicho de paso es una

${ }^{2}$ De hecho, el tema de los talleres literarios constituye un campo de muchas posibilidades para los investigadores interesados en examinar un aspecto fundamental, y no carente de polémicas, del desarrollo de la literatura contemporánea del Ecuador.

${ }^{3}$ Las narradoras antologadas son: Elisa Ayala González (1879-1956), Mary Corylé (1901-1978), Carmen Acevedo Vega (1913), Nela Martínez (1914), Zoila María Castro (1917), Alicia Yánez Cossío (1929), Eugenia Viteri (1930), Lupe Rumazo (1935), Fabiola Solís de King (1936), Argentina Chiriboga (1940), Violeta Luna (1940), Sonia Manzano (1947), Gilda Holst (1952), Liliana Miraglia (1952), Libertad Regalado (1953), Denise Rosales (1954), Jennie Carrasco (1955), Elsy Santillán (1957), Aminta Buenaño (1958), María del Carmen Garcés (1958), Livina Santos (1959), María Eugenia Paz y Miño (1959), Martha Rodríguez (1959), Marcela Vintimilla (1961), Carolina Andrade (1962), Lucrecia Maldonado (1962), Ruth Patricia Rodríguez (1966) y Yanna Hadatty (1969). 
adaptación del modelo de Biblioteca Ayacucho de Venezuela, Donoso Pareja ofrece un estudio introductorio acerca del contenido del libro, datos bio-bibliográficos de las autoras antologadas, algunos juicios de varios críticos sobre la escritura de las mujeres del país, una cronología de las narradoras ecuatorianas que comienza en 1879 y termina en 1996, una bibliografía recomendada, temas para trabajo de los estudiantes y, finalmente, la selección de cincuenta y seis cuentos (dos de cada una de las veintiocho narradoras).

Aparte de la selección de cuentos que sirve como una muestra panorámica de la producción de las narradoras del siglo xx, la introducción de Donoso Pareja incluye explicaciones y comentarios acerca de algunos conceptos de la literatura femenina y feminista, sobre todo en lo que respecta a América Latina. Luego, traza someramente la historia de la prosa de las ecuatorianas en el contexto sociohistórico del Ecuador moderno. Debido al propósito docente de la edición, la introducción se caracteriza por ayudar al lector a ubicar el material antologado de una manera muy general. Aunque los lectores más conocedores del material tratado puedan discrepar con varias de las generalizaciones que hace Donoso Pareja acerca de la escritura y el género como una categoría crítica en el estudio de la literatura, sus comentarios incitan reacciones y, para los lectores e investigadores extranjeros, ofrecen suficiente información para estimular nuevos estudios. Ciertamente, una de las conclusiones más sugererentes de la introducción es que, según Donoso Pareja, "Desde 1980 [...] hasta nuestros días, la presencia de la mujer en la narrativa ecuatoriana es cada vez mayor, a tal punto que son más las escritoras que los escritores aparecidos en este lapso, tanto en calidad como en cantidad” (39).

Del tALler Literario Al Ático FEMinista

Mientras que Donoso Pareja utilizó un enfoque cronológico para ordenar las selecciones de su antología, situando la escritura de las narradoras según los parámetros canónicos de la historia literaria del país (i.e., el liberalismo de comienzos del siglo xx, el realismo social de los años treinta, el inicio de propuestas para actualizar la narrativa ecuatoriana de los años cincuenta, la emergencia de una literatura profundamente marcada por el boom petrolero de los años setenta y el retorno a la democracia de la década del ochenta), Cecilia Ansaldo optó por un esquema más temático al presentar los cuentos de las veintidós narradoras incluídas en Cuentan las mujeres. La clasificación de los veintidós cuentos antologados como realistas, experimentales, feministas, fantásticos y posmodernos se refiere tanto al contenido como a las técnicas discursivas de los textos y, así, se resaltan las mayores tendencias literarias que caracterizan la producción cuentística de las narradoras nacionales del siglo xx. ${ }^{4}$

${ }^{4}$ Las escritoras están agrupadas según el siguiente esquema: CUENTOS REALISTAS -Elisa Ayala (1879-1956), Mary Corylé (1901-1978), Nela Martínez (1914), Alicia Yánez Cossío (1929), Eugenia Viteri (1930); CUENTOS EXPERIMENTALES -Lupe Rumazo (1935), Fabiola Solís de King (1936), Lucrecia Maldonado (1962), Yanna Hadatty (1969); CUENTOS FEMINISTAS Gilda Holst (1952), Marcela Vintimilla (1961), Jennie Carrasco (1955), Carolina Andrade (1962), María Eugenia Paz y Miño (1959); CUENTOS FANTÁSTICOS -Aminta Buenaño (1958), Elsy Santillán (1957), Gabriela Alemán (1968), Sonia María Crespo (1967); CUENTOS POSMODERNOS -Liliana Miraglia (1952), Sonia Manzano (1947), Martha Rodríguez (1959), Solange Rodríguez (1976). 
Aunque Ansaldo no abandona por completo la idea, de Donoso Pareja, de concebir el contexto nacional como una fuerza determinante en la creación literaria, su sistema de identificación rebasa las categorías primordialmente nacionales y evoca pautas críticas que no conocen fronteras geográficas. Es decir, al nivel de las lecturas, Cuentan las mujeres sugiere una retro-alimentación intertextual que logra entrelazar lo nacional con lo latinoamericano. En cierta manera, la organización de esta antología nos remonta a una posible estrategia de valoración crítica capaz de evitar que los estudios sobre la literatura del Ecuador se pierdan en un aislamiento nacional. ${ }^{5}$

Sin duda alguna, Cuentan las mujeres es el producto de las experiencias que Ansaldo ha compartido con el grupo "Mujeres del Ático” de Guayaquil, una especie de colectivo que desde fines de la década del ochenta se ha reunido cada semana para analizar sistemáticamente la situación general de la mujer, primero en el Ecuador y, luego, a nivel mundial. En la introducción a su antología, Ansaldo revela la amplitud de sus conocimientos e inquietudes pertinentes al campo de los estudios de la mujer. Al reconocer, por ejemplo, el peligro de que los lectores consideren una antología de narradoras como una muestra de subliteratura o como un intento de imitar una moda puramente comercial, cuando no políticamente correcta, ella explica que su motivo es "paliar los vacíos de edición y publicación habituales en el reducido ámbito editorial del Ecuador” (10). Después de constatar que su trabajo de antologista no corresponde a la ginocrítica, Ansaldo recuerda

que una política de segregación al momento de hacer una antología puede obedecer estrategias ideológicas, más que de mercado. Entonces, no se busca vender porque la “mujer está de moda” sino que se persiguhe hacer presencia, en todos los frentes posibles, e irradiar los frutos del pensamiento y la sensibilidad femeninos. Hay que llamar la atención, enfatizando la existencia de unas realizaciones estéticas, para que del consumo meramente curioso se derive a la aceptación y valoración de una femenina manera de ver la vida. Cuando se equilibren los niveles de oportunidad, interés y consumo de lo que hacen las mujeres, no habrá necesidad de insistir tanto en la incursión de cuentistas, novelistas y poetas mujeres en la literatura de aquí y de cualquier parte. (13-14)

Curiosamente, aunque Ansaldo comprende la función reivindicativa de su antología, ella todavía manifiesta cierta ambivalencia respecto al proyecto aplicado al contexto ecuatoriano. Es decir, después de reconocer el crecimiento de la producción de las narradoras nacionales, especialmente a partir de 1980, parece titubear ante su propósito cuando comenta: "No tanto como para hablar de presencia significativa, pero sí para

\footnotetext{
${ }^{5}$ No estará de más citar aquí a Angel G. Quintero-Rivera quien señaló recientemente: "La fragmentación latinoamericana en diversos Estados naciones cuando, por otro lado, son numerosos los procesos socio-históricos y culturales que de diversas maneras se comparten, se torna imperativo la necesidad de construir instancias institucionales que, reconociendo la heterogeneidad y respetando la diversidad, faciliten el intercambio de ideas a nivel continental. Aunque el paradigma analítico decimonónico europeo -del Estado nación y la cultura nacional-ha predominado y aún hegemoniza la tradición de las ciencias humanas en nuestro continente, las instancias institucionales de intercambio intelectual regional han jugado un papel fundamental en el desarrollo de las diversas disciplinas académicas" ("LASA y las cambiantes realidades de los estudios latinoamericanistas", LASA Forum, XXXIV, 1 (Spring 2003), 16).
} 
reparar en que las mujeres narradoras se multiplican, con amplio registro temático, ensayando variedad de técnicas, incorporando el humor, el erotismo, la sátira” (17; lo subrayado es nuestro). A nuestro entender, este cuestionamiento de una "presencia significativa" es exagerado y, como ya hemos comentado más arriba, la costumbre de emplear lo numérico como un criterio crítico determinante en la valoración de la literatura ecuatoriana ha conducido a actitudes defensivas y de indiferencia que, a menudo, han imposibilitado una verdadera profundización de los méritos de las letras nacionales. ${ }^{6}$ En efecto, sólo hace falta leer en esta antología cuentos como "La marcha de los batracios" de Lupe Rumazo, "La voz en off” de Gilda Holst, "El extraño éxodo de los Fortunatos o la invasión que salió del mar” de Aminta Buenaño o “Película en dos tiempos” de Liliana Miraglia para comprender que la presencia significativa de las narradoras ecuatorianas (y de los escritores ecuatorianos en general) se ha de medir más allá de las cuantificaciones arbitrarias.

LOS CRíticos TOMAN LA PALABRA DESDE EL EXTERIOR

No pasaremos por alto que la antología de Cecilia Ansaldo fue publicada como parte de la serie de Seix Barral Biblioteca Breve de Editorial Planeta del Ecuador. El prestigio de dicho sello editorial puede considerarse como una afirmación más del valor del material antologado, y del interés cada vez mayor que lo rodea. Sin duda, este interés trasciende lo meramente ecuatoriano ya que en toda Latinoamérica se habla de la producción literaria de las mujeres, muchas de las cuales se han convertido en bestsellers. Por supuesto, este último fenómeno no viene sin controversias en sociedades que se están incorporando aceleradamente a una cultura globalizada en la cual mucho se valora según su rentabilidad. Por lo tanto, el aparente boom de las escritoras latinoamericanas que data desde 1980, apróximadamente, ha sido sometido a no poco escepticismo que, muchas veces, se ha expresado mediante preguntas como: ¿Moda o calidad artística?, ¿expresión propia o prefabricada?, ¿literatura o literatura "light”? Ya se sabe que han existido muchas agendas y diversos intereses detrás de estas reacciones y no es nuestro propósito analizarlas aquí. Sin embargo, la emergencia de las escritoras como una potencia real y/o inventada, según los flujos del mercado y los criterios de quienes la definen, es un hecho innegable y, como tal, ha ratificado la centralidad del tema de las mujeres en todos los campos académicos y no-académicos. Pues, esto es, en parte, la razón por la cual la Editorial de la Universidad de Puerto Rico aceptó publicar Narradoras ecuatorianas de hoy: Una antología crítica, editada por Adelaida López de Martínez y Gloria Da Cunha-Giabbai. Realmente, este

\footnotetext{
${ }^{6}$ Aunque hemos escuchado esta lamentación de una supuesta falta de escritores nacionales en muchas ocasiones, nadie ha podido definir la cantidad necesaria para justificar una "presencia significativa” de la literatura ecuatoriana. Además, ¿cuáles han de ser los fundamentos comparativos empleados en los cálculos? Seguramente, no se ha de esperar que un país con apenas 13 millones de habitantes produzca las mismas cantidades que México, por ejemplo. Pues, la presencia de tales escritores como el Padre Juan de Velasco, Eugenio Espejo, Juan León Mera, Dolores Veintimilla, Zoila Ugarte de Landívar, José de la Cuadra, Jorge Carrera Andrade, Pablo Palacio, Jorge Enrique Adoum, Efraín Jara, Julio Pazos, Alicia Yánez Cossío, Abdón Ubidia, Jorge Velasco Mackenzie, entre muchísimos más, sí es significativa.
} 
proyecto compuesto de catorce estudios críticos sobre la obra de catorce narradoras ecuatorianas, y presentados por trece investigadores radicados fuera del Ecuador (i.e., Estados Unidos, Canadá y Portugal), es insólito. ${ }^{7}$ Para un país como el Ecuador cuya resonancia fuera del ámbito nacional es difícilmente percibida, por lo menos en lo que se refiere a la recepción tradicional de su literatura, es lógico que se pregunte si el interés transnacional generado por sus narradoras, aparentemente tan evidente en la mentada antología crítica, proviene de un compromiso crítico sostenible. La respuesta, claro está, se dará en gran parte con el tiempo, según las futuras publicaciones de las escritoras por un lado, y la medida en que la actual preocupación por la producción de las mujeres a nivel mundial sea más que un mero capricho del mercado, por otro.

Sea lo que sea el destino del boom femenino de nuestros días, lo cierto es que con esta colección de estudios críticos estamos frente a un interesante complemento de las dos antologías ya comentadas. De las catorce narradoras estudiadas, once tienen cuentos en las recopilaciones de Donoso Pareja y de Ansaldo, mientras que otras dos están representadas en la antología de Donoso Pareja. Natasha Salguero es la única que no aparece en las obras anteriores, y es porque hasta la fecha ella ha escrito poesía y una novela en vez de cuentos. Según explica Da Cunha-Giabbai en su introducción, el propósito de su antología crítica es "brindar actualidad y variedad de perspectivas y enfoques para revelar, desde distintos ángulos de observación, la riqueza temática, la heterogeneidad de intereses, la multiplicidad de influencias y de estilos, de perspectivas y de posiciones frente al mundo, que componen el imaginario ecuatoriano femenino” (15). De modo que, mediante un esfuerzo colectivo realizado desde el exterior, esta antología pretende contrarrestar el desconocimiento tradicional que ha mantenido a la literatura ecuatoriana a oscuras, por lo menos en cuanto a su recepción y accesibilidad y, al mismo tiempo, constituye una afirmación más de la "presencia significativa” de las narradoras nacionales.

Básicamente, la colección ofrece catorce comentarios de texto que resaltan los principales temas y técnicas narrativas que caracterizan a cada autora. De interés especial es el cuestionario de cincuentaisiete preguntas al cual cada autora respondió separadamente, y cuyos resultados acompañan cada estudio. Es decir, mientras que los investigadores han utilizado las respuestas bio-bibliográficas para situar y contextualizar mejor los textos analizados, cada cuestionario con sus respuestas respectivas establece un vínculo virtual entre las narradoras y los lectores de la antología. En otras palabras, al tomar en cuenta la novedad que representan las ecuatorianas para un público extranjero, el material de los cuestionarios constituye un contexto informativo y personalizado desde el cual se han de leer los comentarios.

Aunque algunas de las preguntas son repetitivas y un poco trilladas (especialmente las que tratan de las supuestas influencias, las obras preferidas y los temas predilectos), otras han generado respuestas que revelan una tensión constante y contradictoria inherente a la cuestión misma de clasificar a las narradoras según su sexo. Ya se sabe que, de por

\footnotetext{
${ }^{7}$ Las narradoras estudiadas son: Alicia Yánez Cossío, Eugenia Viteri, Carolina Andrade, Aminta Buenaño, Jennie Carrasco Molina, Gilda Holst, Luz Argentina Chiriboga, Sonia Manzano, María Eugenia Paz y Miño, Natasha Salguero, Elsy Santillán Flor, Marcela Vintimilla, María del Carmen Garcés y Liliana Miraglia.
} 
sí, esta discusión no es novedosa. Pero, más allá de la superficie, deja al descubierto lo tenue y lo involucrada que es cualquier referencia a un supuesto imaginario ecuatoriano femenino. Hemos de recordar que al mismo tiempo que se vacila ante la ya mentada "presencia significativa” de la mujer en la literatura por un lado, y las categorías de género, por otro, el Ecuador sigue siendo un país “en ciernes” y, como tal, la construcción de una nueva identidad nacional capaz de reconciliar su condición pluricultural (y hasta plurinacional, según ciertos sectores de la sociedad ecuatoriana), justamente en una época profundamente marcada por la dolarización de la economía y la descentralización del Estado, reclama la presencia y participación activa de las mujeres con intereses particulares y como ciudadanas conscientes de la inseparabilidad de su género, su clase social, su etnia y su nacionalidad. De manera que todo este conjunto de eventos e influencias pone de relieve el contexto sociohistórico en que se ha de estudiar y valorar a las narradoras actuales (y la literatura ecuatoriana en general).

Reconocemos que los comentarios de texto publicados en Narradoras ecuatorianas de hoy: Una antología crítica, junto con el manejo de los cuestionarios señalados, solamente raspan la superficie de las posibilidades de investigación que implica cualquier aproximación que pretenda abarcar el lugar complejo que ocupa la mujer ecuatoriana en las mútliples facetas de la historia nacional y latinoamericana. Sin embargo, la principal virtud de esta labor crítica e investigativa radica en ofrecer a los lectores una primera muestra amplia de materiales que puede conducir a una futura elaboración sistemática de lecturas y análisis y, así, superar la naturaleza esporádica y discontinua que caracteriza gran parte de la crítica tradicional dedicada a la literatura ecuatoriana llevada a cabo desde el exterior. Esta meta será especialmente factible si se leen conjuntamente las tres antologías comentadas aquí para, de esta manera, establecer una perspectiva integral del material. ${ }^{8}$

\section{ConCLusiones}

No ha de extrañarnos que estas antologías hayan destacado una variada muestra de cuentos escritos por casi una treintena de narradoras ecuatorianas, ya que este género literario tiene una larga y rica historia en la literatura nacional. Sin embargo, todavía quedan por estudiarse más a fondo, y desde diversas perspectivas, la producción de cuentos de las ecuatorianas. Al mismo tiempo, hemos de recalcar que hay también mucho que investigar acerca del aporte de las mujeres a la poesía, al teatro, al cine y a la crítica literaria, entre muchos otros campos, ora socioculturales ora sociopolíticos. Como ya hemos constatado, las particularidades que apuntan a conceptos de género y que piden una atención especial y especializada, no han de sugerir necesariamente una separación

\footnotetext{
${ }^{8}$ De hecho, si encontramos una limitación en esta antología, es la aparente falta de diálogo textual entre los diversos estudios (y estudiosos). Es decir, como comentarios de texto, hay una tendencia a descontextualizar cada análisis y, así, no trascender realmente los contornos individuales de la producción de cada autora, por un lado, y el cuestionario como principal fuente de referentes sociales, por otro. Con la excepción de la introducción que trata de unir el material y, de esta manera, darle cierta coherencia, este esfuerzo colectivo sigue siendo más aparente que real.
} 
esencialista de la producción de las mujeres y la de los hombres. Ya se sabe que toda sociedad pluralista está destinada a negociar continuamente sus diferencias, y la decisión de enfocar a las mujeres como una categoría propia -entre otras categorías igualmente pertinentes-constituye un componente medular de ese proyecto heterogéneo y pluricultural que es la reconstrucción de las identidades nacionales.

Finalmente, se recordará que hace años, Gayatri Spivak declaró que los subalternos (una categoría que incluye a las mujeres) no podían hablar dentro de sociedades que todavía no se habían liberado de los imperialismos de Occidente. A riesgo de simplificar su argumentación que servía, en parte, como una refutación de algunas ideas de Foucault y de Deleuze sobre la (re)presentación del Otro, que ella consideraba una práctica engañosa de apropiación, quisiéramos insistir que, pese a las innumerables desigualdades e injusticias tan presentes en la historia de los diversos colonialismos, en lo que se refiere a América Latina, y a la región andina en particular, los subalternos han estado hablando con múltiples registros simbólicos y contestatarios desde el mismo día en que Atahuallpa tiró la Biblia (es decir, la palabra de Dios que el Inca no logró oír) a los pies del Padre Valverde, expresando con gran elocuencia un acto de afirmación cultural. Traemos esta breve referencia histórica a colación aquí porque nos recuerda que muchas veces los llamados dueños de la palabra -los mismos que se han autodeclarado responsables de redactar e interpretar sus historias oficiales- no han sabido (o querido) escuchar a los subalternos. Es precisamente en este sentido que consideramos necesario leer las antologías preparadas por Miguel Donoso Pareja, Cecilia Ansaldo, Adelaida López de Martínez y Gloria Da Cunha-Giabbai. Vistas como parte de una larga historia de revisiones y revaloraciones de supuestos silencios y ausencias, se comprenderá que el material recogido es un testimonio vital que sigue convocándonos a (re)descubrir las múltiples formas y tonalidades de la voz de las escritoras ecuatorianas quienes, a su vez, son representativas de cualquier grupo destinado a desarticular su marginalidad. En efecto, mientras las mujeres del Ecuador esperan que se las escuche, las tres antologías que nos han ocupado en este ensayo comprueban que las mujeres también cuentan en el Ecuador.

Bibliografía

Ansaldo, Cecilia. (Editora). Cuentan las mujeres: Antología de narradoras ecuatorianas. Quito: Editorial Planeta del Ecuador, 2001.

Donoso Pareja, Miguel. (Editor). Antología de narradoras ecuatorianas. Quito: Libresa, 1997.

“La mujer en la narrativa ecuatoriana,” Revista de Hoy (10 de julio de 1983): 4.

López de Martínez, Adelaida y Gloria Da Cunha-Giabbai. (Editoras). Narradoras ecuatorianas de hoy. San Juan: Editorial de la Universidad de Puerto Rico, 2000.

Quintero-Rivera, Angel G. "LASA y las cambiantes realidades de los estudios latinoamericanistas”. LASA Forum XXXIV/1 (Spring 2003): 16.

Showalter, Elaine. (Editora). The New Feminist Criticism: Essays on Women, Literature, and Theory. New York: Pantheon Books, 1985. 